\title{
Phase-coherent THz-wave demodulation with a photomixer
}

\author{
Florin Lucian Constantin ${ }^{1, *}$ \\ ${ }^{1}$ Laboratoire PhLAM, CNRS UMR 8523, 59655 Villeneuve d'Ascq, France
}

\begin{abstract}
The nonlinear electrical response from a low temperature grown GaAs photomixer driven with the optical beat of two continuous-wave lasers allows frequency mixing between radiofrequency and THzwaves. Phase-sensitive demodulation of the signal from a modulated THz-wave is theoretically and experimentally demonstrated using a photomixer electrically driven with an alternative voltage at the modulation frequency.
\end{abstract}

\section{Introduction}

The Terahertz photonics research has applications in various domains including biological imaging, remote sensing, high-speed telecommunications, and security screening [1]. The optical frequency down-conversion (photomixing) was exploited for continuous-wave (cw) $\mathrm{THz}$ generation using the optical beat of two cw lasers and an ultrafast photoconductive antenna [2]. The optical frequency control enabled compact, room-temperature operated, cost-effective and low-power consuming $\mathrm{THz}$ photomixing systems with narrow spectral linewidth, wide frequency tunability, high dynamic range and high signal-to-noise ratio. The modulation of the $\mathrm{THz}$ waves, critical parameter for high-speed wireless telecommunications, was demonstrated through various approaches [3]. Particularly, the nonlinear electrical conduction in a low temperature grown GaAs (LTGGaAs) photomixer was exploited for direct current modulation of the photomixing signal [4]. This contribution addresses theoretically and experimentally the demodulation of a THz-wave using the photomixing approach. The LTG-GaAs photomixer was exploited for the detection of a modulated THz-wave [5]. When the photomixer is electrically driven with an alternative voltage oscillating at the modulation frequency of the THz-wave, the phase-sensitive DC response signal of the photomixer indicates the demodulation of the THz-wave.

\section{Results}

The electrical conduction in the photomixer is modeled by unidimensional carrier transport in a thin LTG-GaAs layer. Two laser beams, carrying the same optical power $\mathrm{P}$ are detuned by an angular frequency $\omega$ around the mean angular frequency $\bar{\sigma}$, are spatially superposed and enter at normal incidence in the photoconductor. The current density is expressed as:

$$
j(t)=\frac{2 e \eta P}{\hbar \varpi A d}\left[\begin{array}{c}
\tau_{n}(E) v_{n}(E)\left(1+\frac{\omega \tau_{n}(E) \sin (\omega t)+\cos (\omega t)}{1+\left(\omega \tau_{n}(E)\right)^{2}}\right) \\
+\tau_{p}(E) v_{p}(E)\left(1+\frac{\omega \tau_{p}(E) \sin (\omega t)+\cos (\omega t)}{1+\left(\omega \tau_{p}(E)\right)^{2}}\right)
\end{array}\right]
$$

The superposed laser beams are focused on a photoconductive area $\mathrm{A}$, and absorbed within a depth $\mathrm{d}$ with a quantum efficiency $\eta$. The carrier lifetimes $\tau_{\mathrm{n}, \mathrm{p}}(E)$ depend on the applied electric field [6]. The dependences are described here with linear functions that take into account the carrier heating:

$$
\tau_{n, p}(E) \approx \tau_{0 n, 0 p}\left[1+3 \lambda e E /\left(2 k_{B} T\right)\right]
$$

in function of the Boltzmann constant $k_{B}$, the electron charge $e$, the room temperature $T=300 \mathrm{~K}$, the low-field carrier lifetimes $\tau_{0 n, 0 p}$ and the distance $\lambda$ between the Coulomb barriers in the LTG-GaAs photoconductor. The carrier drift velocities have a nonlinear dependence on the electrical field $[7,8]$, expressed as:

$$
v_{n, p}(E)=v_{s n, s p} /\left[1+v_{s n, s p} /\left(\mu_{0 n, 0 p} E\right)\right]
$$

with the saturation velocities $v_{s n, s p}$ and the low-field mobilities $\mu_{0 n, 0 p}$ of the electrons and holes.

The total electric field in the photoconductor may be expressed as:

$$
\begin{aligned}
& E(t)=E_{D C}+E_{R F} \cos \left(\omega_{R F} t+\phi_{R F}\right) \\
& +\sum_{n} E_{T H z, n} \cos \left(\left(\omega_{T H z}+n \omega_{R F}\right) t+\phi_{T H z, n}\right)
\end{aligned}
$$

the sum between a bias electric field $E_{D C}$ and a radiofrequency electric field (amplitude $E_{R F}$, angular frequency $\omega_{R F}$, phase $\phi_{R F}$ ) from an alternative voltage applied on the photomixer electrodes. The last terms are the spectral components of a $\mathrm{THz}$ electric field (amplitudes $E_{T H z, n}$ angular frequencies $\omega_{T H z}+n \omega_{R F}$, 
phases $\phi_{T H z, n}$ ) that is induced by a THz-wave modulated at $\omega_{R F}$ which is detected with the photomixer. The electrical nonlinearity arising from $v_{n, p}(E)$ and $\tau_{n, p}(E)$ dependences enables frequency mixings between the THz-wave spectral components and the radiofrequency in the LTG-GaAs photoconductor. The amplitudes and phases of the spectral components of the current density are analytically calculated with eq. (1-4), using the photomixer parameters $\mathrm{d}=2 \mu \mathrm{m}, \mathrm{A}=12.6 \mu \mathrm{m}^{2}, \hbar \varpi / e=1.5$ $\mathrm{V}, \eta=0.68$, the LTG-GaAs photoconductor parameters from refs. [6-8] and the modulation parameters. Particularly, the modulated $\mathrm{THz}$-wave generates a spectral component of the current density at $\omega_{R F}$ that can be demodulated by coherent mixing with the radiofrequency signal at $\omega_{R F}$ applied on the phomixer electrodes.
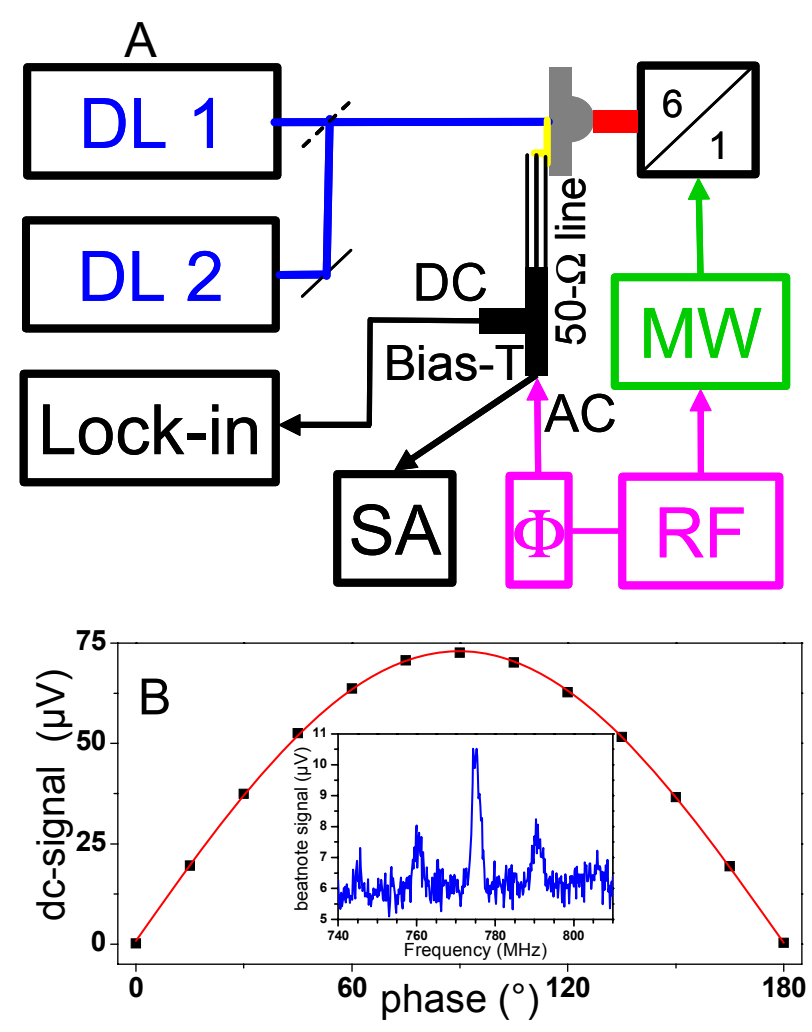

Fig. 1. A. The experimental setup. The signal from the radiofrequency synthesizer RF is used to modulate the THzwave and, after application of an adjustable phase offset, is coupled to the photomixer. B. Phase-sensitive dependence of the demodulated signal. Inset : Spectrum of the heterodyne detection signal of a THz-wave at $102.072 \mathrm{GHz}$ modulated at $15 \mathrm{MHz}$. Optical beat power $2 \times 14 \mathrm{~mW}$. RBW=1 MHz, sweep time $4 \mathrm{~ms}, 16$ video averages.

The experiment (Fig. 1.A) is based on a LTG-GaAs photomixer with a log-spiral antenna driven by the optical beat of two extended cavity diode lasers (DL1 and DL2) emitting at $820 \mathrm{~nm}$. The extremities of the antenna are connected to a coaxial microwave line addressed with a bias-T. The DC path is used to measure the response from the photomixer using a lock-in detection technique (Lock-in). The AC path is used to record the heterodyne down-conversion signals with a microwave spectrum analyser (SA) or, alternatively, to apply direct-current modulation on the photomixer with a RF synthesizer (RF). A 75-110 GHz electronic frequency multiplier driven by a microwave synthesizer (MW) is used to generate a THz-wave which is focused on the photomixer antenna with a silicon lens. All synthesisers and the spectrum analyser are phasereferenced on a $10 \mathrm{MHz}$ GPS-disciplined quartz oscillator.

A multifrequency $\mathrm{THz}$-wave is generated by modulating MW with a $15 \mathrm{MHz}$ signal provided by RF. The spectrum of the THz-wave is down-converted using the heterodyne detection scheme with the photomixer and recorded with SA. The spectral components, spaced by the modulation frequency, are shown in the inset of Fig. 1.B. The next step is to demodulate the $\mathrm{THz}$ waveform by exploiting a phase-delayed signal from RF (amplitude $1.5 \mathrm{~V}$, frequency $15 \mathrm{MHz}$ ) that is capacitively coupled to the photomixer. For sensitive detection, RF is $100 \%$ amplitude modulated at $1 \mathrm{kHz}$ and the dc-signal in the photomixer circuit is recovered with a lock-in amplifier. The Fig. 1.B presents the dependence of the dc-signal in function of the relative phase between the THz-wave modulation signal and signal applied to the photomixer. The sinusoidal dependence demonstrates demodulation of the THz-wave with the photomixer. The photomixing approach enables integration of the functions of the optoelectronic detection and the electrically-driven demodulation in a single photonic circuit for $\mathrm{THz}$ signal processing.

\section{References}

1. S.S. Dhillon et al., J. Phys. D 50, 043001 (2017)

2. E.R. Brown et al., Appl. Phys. Lett. 66, 285-287 (1995)

3. Z.T. Ma et al., Research Vol. 2019, 6482975 (2019)

4. F.L. Constantin, Proc. of SPIE Vol. 9894, 98941K (2016)

5. F.L. Constantin, IEEE J. Quantum Electr. 47, 14581462 (2011)

6. N. Zamdmer and Q. Hu, Appl. Phys. Lett. 75, 23132315 (1999)

7. E.R. Brown, Appl. Phys. Lett. 75, 769-771 (1999)

8. E.R. Brown et al., Appl. Phys. Lett. 64, 3311-3313 (1994) 